\title{
Photobiomodulation on KATP Channels of Kir6.2-Transfected HEK-293 Cells
}

\author{
Fu-qing Zhong, ${ }^{1}$ Yang $\mathrm{Li}^{2}$ and Xian-qiang $\mathrm{Mi}^{1,3,4}$ \\ ${ }^{1}$ College of Medical Device and Food Engineering, University of Shanghai for Science and Technology, Shanghai 200093, China \\ ${ }^{2}$ CAS Key Laboratory of Receptor Research, Shanghai Institute of Materia Medica, Chinese Academy of Sciences, \\ Shanghai 201203, China \\ ${ }^{3}$ Key Laboratory of System Biology, Chinese Academy of Sciences, Shanghai 201210, China \\ ${ }^{4}$ Laboratory of System Biology, Shanghai Advanced Research Institute, Chinese Academy of Sciences, Shanghai 201210, China
}

Correspondence should be addressed to Yang Li; liyang@simm.ac.cn and Xian-qiang Mi; mixq@sari.ac.cn

Received 9 January 2014; Accepted 15 February 2014; Published 6 April 2014

Academic Editor: Timon Cheng-Yi Liu

Copyright (c) 2014 Fu-qing Zhong et al. This is an open access article distributed under the Creative Commons Attribution License, which permits unrestricted use, distribution, and reproduction in any medium, provided the original work is properly cited.

\begin{abstract}
Background and Objective. ATP-sensitive potassium (KATP) channel couples cell metabolism to excitability. To explore role of KATP channels in cellular photobiomodulation, we designed experiment to study effect of low intensity 808 nm laser irradiation on the activity of membrane KATP channel. Study Design/Materials and Methods. Plasmids encoding Kir6.2 was constructed and heterologously expressed in cultured mammalian HEK-293 cells. The patch-clamp and data acquisition systems were used to record KATP channel current before and after irradiation. A laser beam of Ga-As $808 \mathrm{~nm}$ at $5 \mathrm{~mW} / \mathrm{cm}^{2}$ was used in experiments. A one-way ANOVA test followed by a post hoc Student-Newman-Keuls test was used to assess the statistical differences between data groups. Results. Obvious openings of KATP channels of Kir6.2-transfected HEK-293 cells and excised patches were recorded during and after low intensity $808 \mathrm{~nm}$ laser irradiation. Compared with the channels that did not undergo irradiation, open probability, current amplitude, and dwell time of KATP channels after irradiation improved. Conclusions. Low intensity 808 nm laser irradiation may activate membrane KATP channels of Kir6.2-transfected HEK-293 cells and in excised patches.
\end{abstract}

\section{Introduction}

It has been demonstrated that irradiation of specific infrared wavelengths is able to penetrate scalp and skull and can reach superficial layers of the cerebral cortex [1]. A recent research confirmed that transcranial laser stimulation (TLS) with low intensity near-infrared laser (NIR) can modulate the excitability of the motor cortex [2]. As constant fluctuation of excitability is a fundamental characteristic of neurons in both central and peripheral nervous system and the homeostatic regulation of neuronal excitability is mainly effected through the mechanisms involved in maintenance of the membrane potential, these findings give further insights into the mechanisms of TLS effects in the human cerebral cortex.

ATP-sensitive potassium (KATP) channels are widely expressed in cytoplasmic membranes of neurons and are the marker of cellular energy metabolism [3]. They link the membrane potential to the metabolic state of the cell and can regulate neuronal excitability. KATP channels may mediate a potential neuroprotective role which may be achieved by membrane hyperpolarization and reduction of excitability in response to hypoxia, ischemia or metabolic stress. Using UV photoirradiation stimulation, the effects of cell stress on KATP channels were studied and the linkage between KATP channels and cellular stress has been confirmed [4]. Nevertheless, there has been no study published so far on effects of low intensity infrared laser irradiation on KATP channel.

Here, we designed experiment to study effect of low intensity infrared laser irradiation on the activity of KATP channels. Molecular cloning of KATP channels was constructed into plasmid and was overexpressed in HEK293 cells. By using patch clamp electrophysiology techniques, we investigated the KATP channels activities under low intensity $808 \mathrm{~nm}$ laser irradiation. We found that infrared low intensity laser irradiation activated KATP channels of Kir6.2-transfected HEK-293 cells and in excised patches directly. 


\section{Materials and Methods}

2.1. Construction of Plasmids Encoding Kir6.2. A truncated form of the Kir6.2 subunit, Kir6.2 $\Delta$ C 35, was constructed using a PCR-based site-directed mutagenesis kit, ExSite (Stratagene, CA, USA), in a mammalian expression vector, pcDNA3.1 (Invitrogen, CA, USA). The Kir6.2 $\Delta$ C35 sequence was also subcloned into a yeast expression vector, $\mathrm{pYES} 2 / \mathrm{NT}$ (Invitrogen), containing a GAL1 promoter for high-level inducible protein expression by galactose and repression by glucose, for expression with a (His) ${ }_{6}$-Xpress tag at its $\mathrm{N}$-terminus for affinity purification and antibody recognition. Kir6.2 $\Delta$ C35 without SUR subunits expresses functional ATP-sensitive channels in mammalian and yeast cells. For simplicity, in this study Kir6.2 $\Delta$ C 35 is referred to as Kir6.2.

2.2. Heterologous Expression in Cultured Mammalian Cells (HEK-293 Cells). The pcDNA3.1-Kir6.2 vector was introduced into HEK-293 cells. A HEK-293 cells cell line was maintained in continuous culture. Kir6.2 in mammalian expression vectors was transiently transfected with an Effectene transfection kit (Qiangen, Hiden, Germany). The channels were expressed in the absence of sulfonylurea receptor (SUR) regulatory subunits. Channel expression usually peaked 36-48 hours after transfection, at which time the cells were used for patch-clamp experiments.

2.3. Chemicals. Inside-out single or multiple channel currents were recorded with an intracellular solution containing $140 \mathrm{mM} \mathrm{KCl}, 5.5 \mathrm{mM}$ HEPES, and 2 mM EGTA (pH 7.4) and an extracellular solution containing $130 \mathrm{mM} \mathrm{NaCl}, 10 \mathrm{mM}$ $\mathrm{KCl}, 1.8 \mathrm{mM} \mathrm{CaCl} 2,0.48 \mathrm{mM} \mathrm{MgCl} 2$, and $5.5 \mathrm{mM}$ HEPES ( $\mathrm{pH}$ 7.4). In some experiments, $\mathrm{NaCl}$ in the extracellular solution was replaced with $130 \mathrm{mM} \mathrm{KCl}$.

2.4. Electrophysiological Recording. The patch-clamp and data acquisition systems used were Axopatch 200B amplifier with a Digidata 1440 A and pCLAMP10.2 software (Axon Instruments, USA) running on a PC. Solution changes in the bath (membrane side of channel in excised patches) were achieved within $100 \mathrm{~ms}$ by means of a rapid solution exchange system (RSC-200, Bio-Logic Science Instruments, Claix, France). All current recordings were filtered at $1 \mathrm{kHz}$ and digitized at $10 \mathrm{kHz}$. Outward currents are shown as upward deviations from the closed level. The current traces were plotted after being manually corrected for baseline shift and filtered with a low-pass digital filter at a cut-off frequency of $500 \mathrm{~Hz}$. Unless specified, currents were recorded at a membrane potential of $0 \mathrm{mV}$. B150-86-10HP Glass Micropipette and P97 Flaming/Brown Micropipette Puller (Sutter Instrument Company, USA). The pipettes required 1 2 micron tip and $5 \sim 10 \mathrm{M} \Omega$ of resistance.

2.5. Laser Irradiation. A laser beam of Ga-As $808 \mathrm{~nm}$ from semiconductor laser (SHENZHEN LAMPLICSCIENCECO.LTD) was used in experiments. The laser power density was kept constant at $5 \mathrm{~mW} \mathrm{~cm}{ }^{-2}$ measured by power meter (Lasercheck, Coherent). The distance is $15 \mathrm{~cm}$; the angle is $75^{\circ}$.

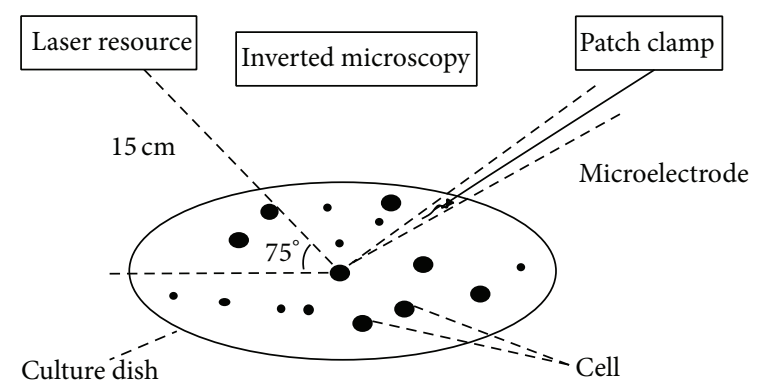

FIGURE 1: The sketch of laser irradiation system.

The laser beam was delivered to samples in dishes with the $3 \mathrm{~mm}$ diameter of irradiation spot (Figure 1). The phenomena of cells being irradiated by red light from laser system can be observed under the inverted microscopy.

2.6. Analysis of Single-Channel Data. Direct $P_{O}$ (opening probability) measurement is used as a quick indicator of channel activity. The value of $P_{\mathrm{O}}$ has a biological interpretation. For cells that demonstrate an action potential, the action potential is the sum of the single-channel current through many ion channel. The $P_{O}$ was measured over a large number of channels. $P_{O}=t_{O} / T, T$ is the total recording time and $t_{O}$ is the open time. In macropatch recordings containing more than five active channels, the apparent open probability $\left(N P_{O}\right)$ was used, where $N$ is the number of channels, an unknown parameter in a macropatch; then $\left(N P_{O}\right)=T_{O} / N T$, and $T_{O}=\sum_{L=1}^{N} t_{O L}, L$ is the level of channel open; $L=1,2$, $3, \ldots . t_{\mathrm{OL}}$ is the open time of different levels. Although ion channels state transits suddenly, the dwell time of an ion channel at a given conductance level is long enough to be observed with electrophysiological recording techniques. The investigator can identify channel transitions (openings and closings) in the recorded data. The result is an "event table" describing each transition of an idealized data trace. This process is also called "event detection." According to the opening probability resulting from ligand concentration, the doseresponse curves can be made. In general, the horizontal axis uses a logarithmic scale $(\log [A])$, and the vertical axis indicates open probability $\left(P_{\mathrm{O}} / P_{\max }\right) ; P_{\max }$ is the maximal open probability. The concentration of ATP ([ATP]) producing half-maximal inhibition $\left(\mathrm{IC}_{50}\right)$ and the Hill coefficient $(\mathrm{H})$ were obtained by fitting the measured open probability data with the expression: $P_{\mathrm{O}} / P_{\max }=1-A^{H} /\left(A^{H}+\mathrm{IC}_{50}{ }^{H}\right)$.

2.7. Statistical Analysis. Experimental data are presented as Mean \pm S.E.M. A one-way ANOVA test followed by a post hoc Student-Newman-Keuls test was used to assess the statistical differences between data groups. Student's $t$-test was used wherever two groups of data were compared.

\section{Results}

3.1. Activation of KATP Channels. For the HEK-293 cells without KATP channels, no current was recorded in attached 

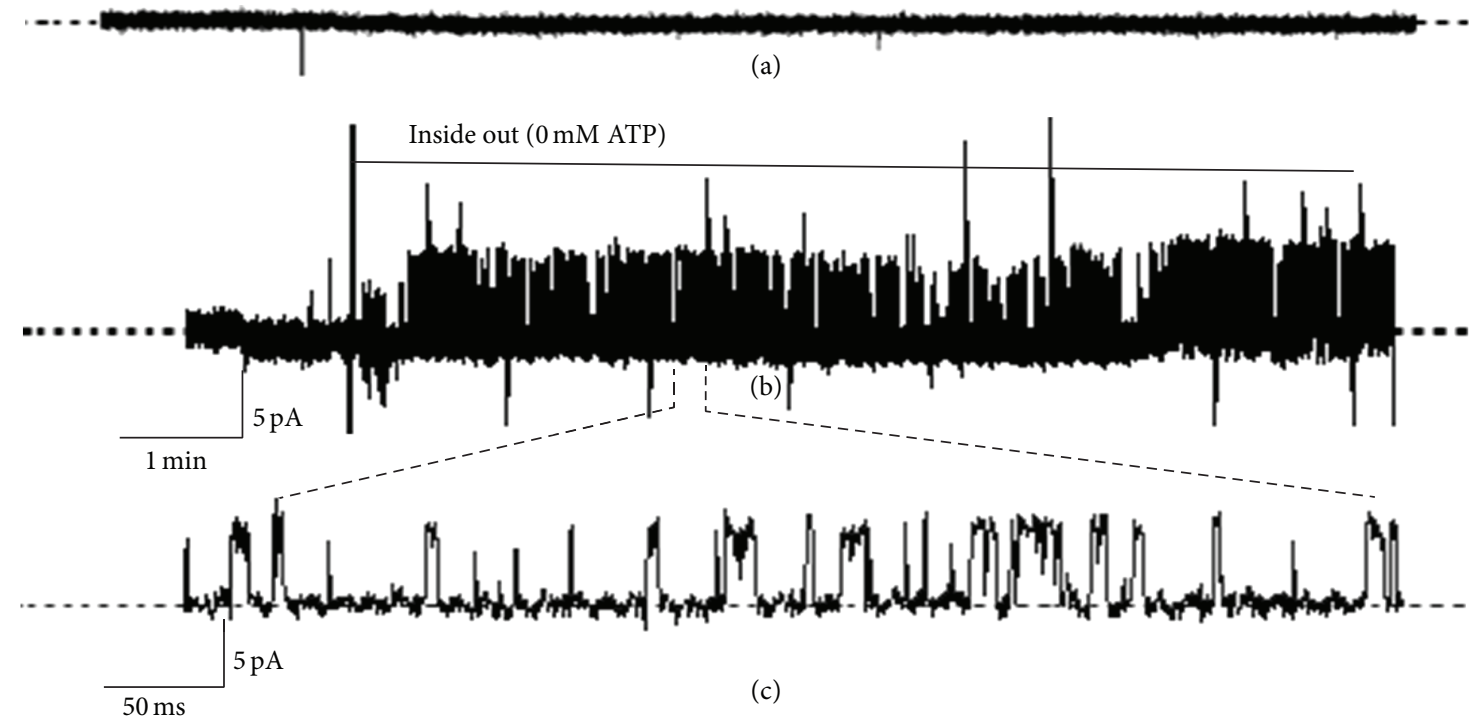

Figure 2: Activation of potassium channels in Kir6.2-transfected HEK-293 cells. (a) HEK-293 cells not expressing Kir6.2. No current was recorded in attached and inside-out patch clamp. (b) HEK-293 cells expressing Kir6.2. The patch membrane was excised at the time marked inside out. The cell was bathed in extracellular solution without ATP, and the patch membrane was held at $0 \mathrm{mV}$ throughout the recording period. (c) A specific time domain was displayed.

and inside-out patch clamp (Figure 2(a)). A significant channel current was recorded from the patch membrane of Kir6.2transfected HEK-293 cells (the cell was bathed in extracellular solution without ATP, the patch membrane was held at $0 \mathrm{mV}$ throughout the recording period, and the patch membrane was excised at the time marked inside out). Channel current of a specific time domain was displayed in Figure 2(c).

3.2. Effect of ATP on the KATP Channels. As shown in the recording trace in Figure 3(a), the patch membrane was excised from the cell, and the sensitivity of the channels to inhibition by ATP was measured. Upon excision of the patch membrane into a solution with an ATP concentration ([ATP]) of $1 \mu \mathrm{M}$, outward KATP channel currents were recorded in all of the successful patch excisions. The ATP sensitivity was obtained by varying [ATP] in the intracellular solution. Changes in [ATP] were made by a series of both increasing and decreasing concentration steps of 10-20 s (this protocol has been shown to be effective at minimizing possible errors caused by run-down of the channels). Relationships between concentration and the ATP inhibition of KATP channels were shown in Figure 3(b).

3.3. Effect of Photobiomodulation on the Opening of KATP Channel. $808 \mathrm{~nm}$ laser irradiation on KATP channels was examined in HEK-293 cells expressing Kir6.2. Figure 4 illustrates an experimental recording of a patch containing multiple channels initially recorded in the cell-attached configuration on a HEK-293 cells expressing Kir6.2. After establishment of the cell-attached patch-clamp configuration, the cell was exposed to a spectrum of $5 \mathrm{~mW} / \mathrm{cm}^{2}$ at $808 \mathrm{~nm}$ via the microscope; the exposure lasted 1-2 min, obvious openings of KATP channels were recorded during and after irradiation, and the current amplitude improved (Figure 4(a)). In 8 cells tested, 5 exhibited clear openings of KATP channels after the irradiation was started. After inside out, the ATP sensitivity was obtained by varying [ATP] in the intracellular solution. Changes in [ATP] were made by a series of both increasing and decreasing concentration steps of 10-20 s. In these experiments, laser irradiation was applied to excised patch membranes while KATP channel currents were recorded in the inside-out patch-clamp configuration during perfusion with intracellular solution containing modulators of channel activity. Irradiation induced a significant increase in the maximal open probability of the active channels. Channel current of a specific time domain was displayed in Figure 4(b).

3.4. Effect of Photobiomodulation on Single KATP Channel. The photobiomodulation on KATP channels was further analyzed kinetically in two patches that contained only a single active channel. An experimental recording of the photobiomodulation on single KATP channels was illustrated. In the example given in Figure 5, a Kir6.2 channel was active from the beginning of the experiment when the excised patch was perfused without ATP. It shows that open probability, current amplitude, and dwell time of KATP channel improved after the irradiation.

Compared with the channels that did not undergo irradiation, the current amplitude and dwell time of KATP channel improved after irradiation (Table 1). The improvement of current amplitude and dwell time distributions after irradiation 


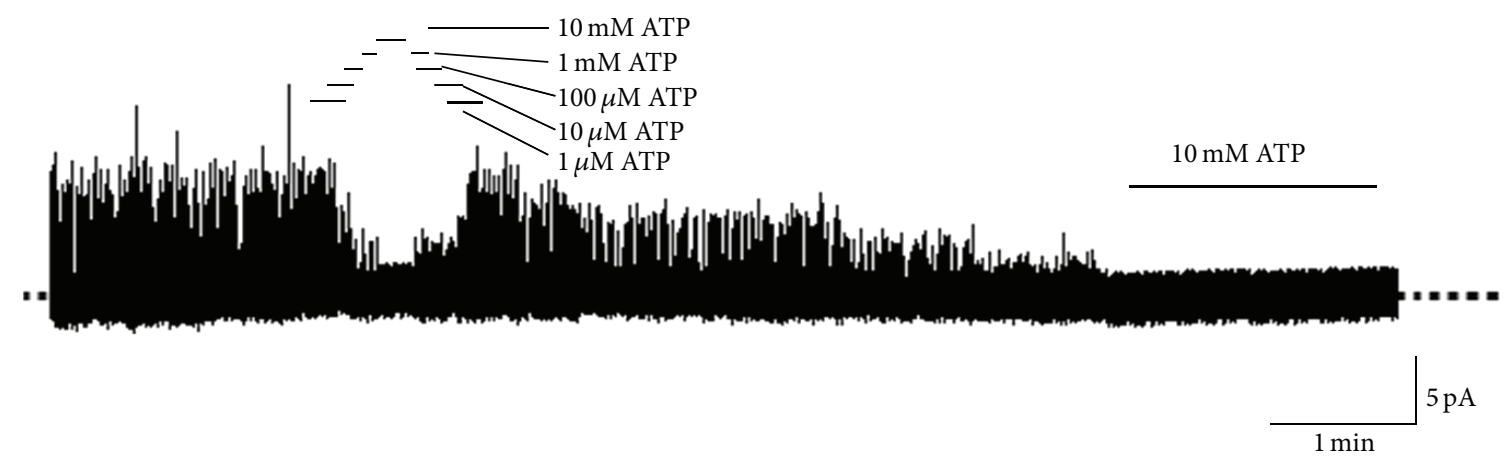

(a)

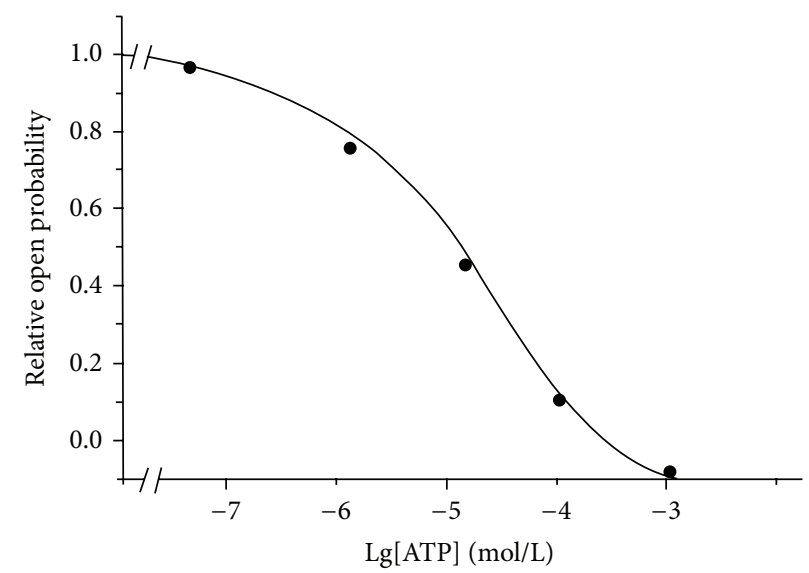

(b)

Figure 3: Different concentrations of ATP on the KATP channel effects. (a) The potassium current increased in response to 0 mM ATP (inside out) and decreased in response to $10 \mathrm{mM}$ ATP. No open channel event in $1 \mathrm{mM}$ ATP was observed. HEK-293 cells expressing Kir6.2 was bathed in extracellular solution, and the patch membrane was held at $0 \mathrm{mV}$ throughout the recording period. (b) Relationships between concentration and the ATP inhibition of KATP channels.

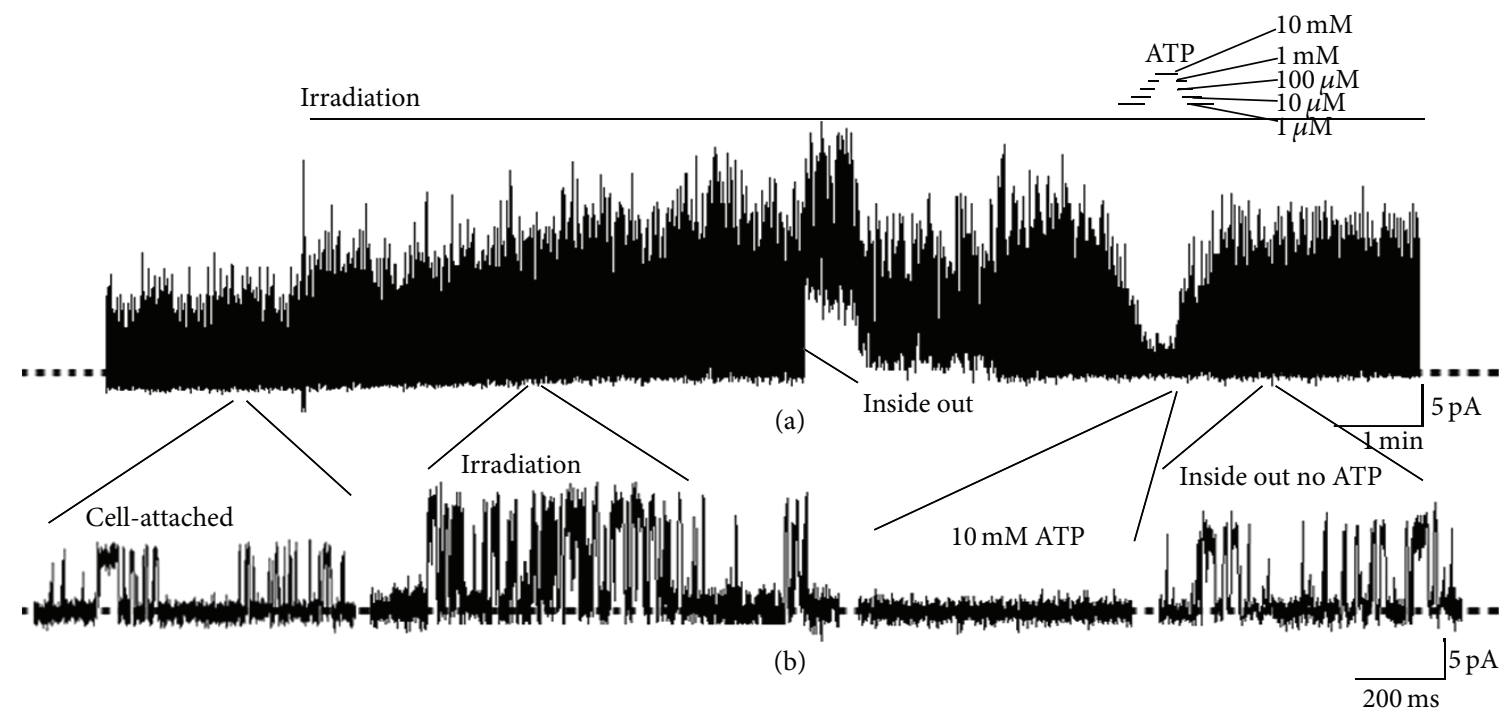

FIGURE 4: Opening of KATP channels by photobiomodulation in a Kir6.2-transfected HEK-293 cell. The current was initially recorded in the cell-attached configuration. The patch membrane was excised at the time marked Inside out. The cell was bathed in extracellular solution, and the patch membrane was held at $0 \mathrm{mV}$ throughout the recording period. ATP was applied at the concentrations indicated by the bars above the current trace. Irradiation indicates the period during which the patched cell was exposed to photobiomodulation (see text for details of photobiomodulation treatment). In this and subsequent figures, the dotted line through the current recording indicates the closed channel level. 


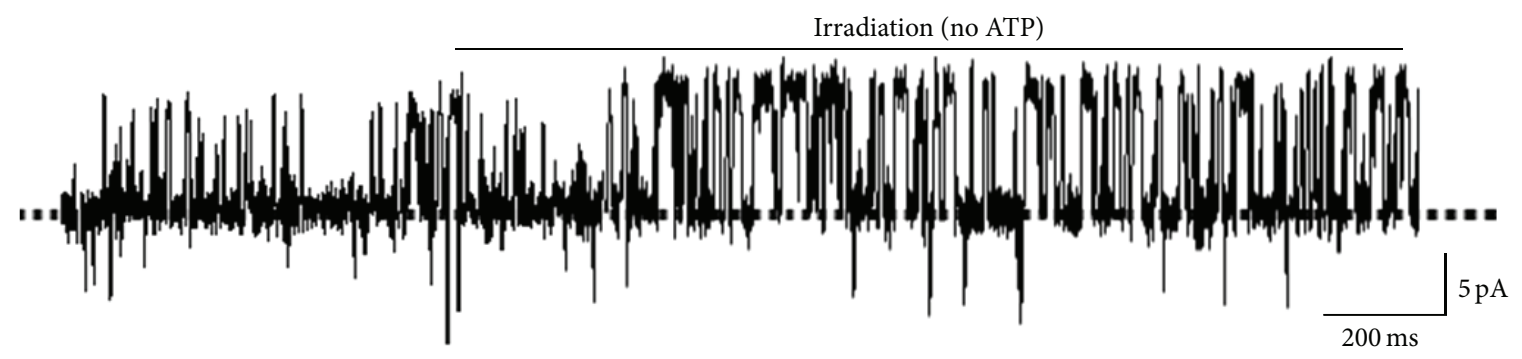

FIGURE 5: Photobiomodulation-induced activation of single-channel current recorded from a Kir6.2-transfected HEK-293 cell. The membrane was held at $0 \mathrm{mV}$. The open probability, current amplitude, and dwell time of KATP channel have changed after irradiation.

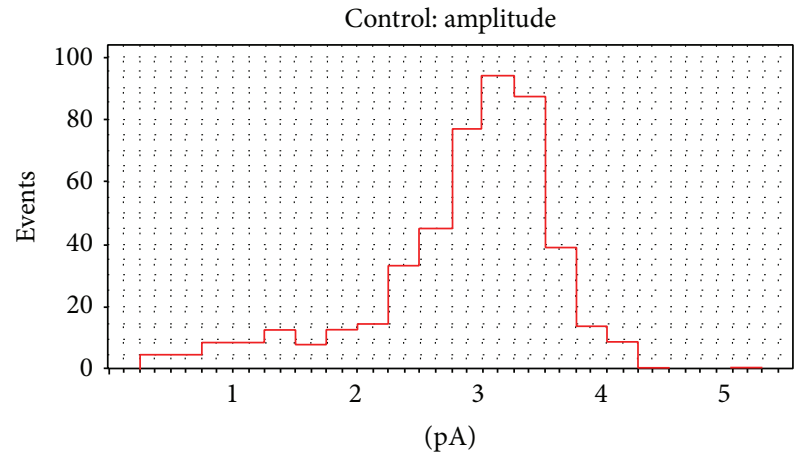

(a)

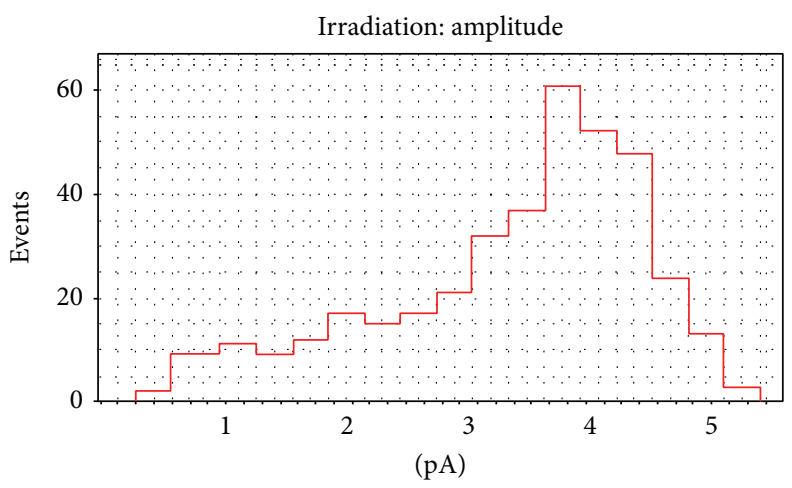

(c)

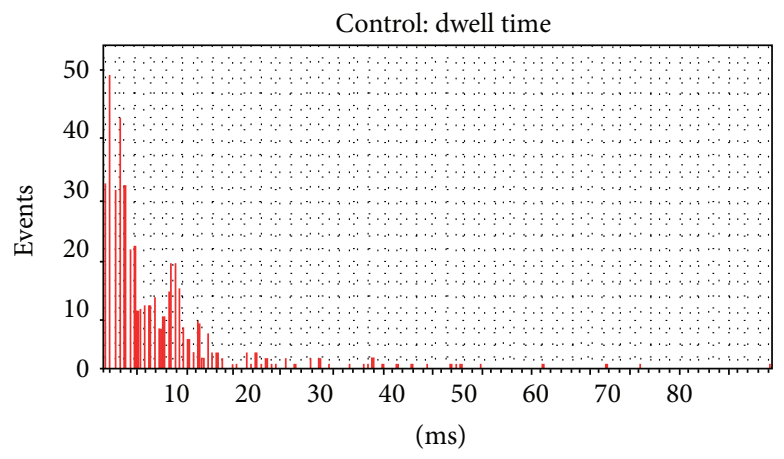

(b)

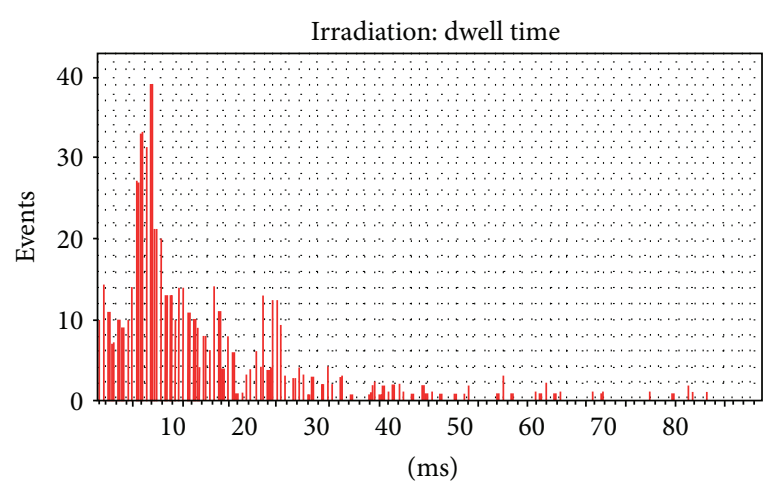

(d)

FIGURE 6: Photobiomodulation on amplitude and dwell time. The Kir6.2-transfected HEK-293 cell was bathed in extracellular solution without ATP, and the patch membrane was held at $0 \mathrm{mV}$ throughout the recording period.

TABLE 1: Effects of irradiation on closed and open time distributions.

\begin{tabular}{lccc}
\hline & Amplitude & Dwell time & Count \\
\hline Control & $3.1583 \pm 0.5371$ & $6.4813 \pm 3.3475$ & 972 \\
Irradiation & $3.9406 \pm 0.6147^{* *}$ & $10.7639 \pm 4.7935^{* *}$ & 1047 \\
\hline
\end{tabular}

${ }^{* *} P<0.01$. Control: before irradiation, irradiation: after irradiation.

can also be seen from the histograms from the single-channel current of patch excised from a Kir6.2 channel (Figure 6).

Maximal open probability $\left(P_{\max }\right)$ has statistically been confirmed to increase after irradiation. Under control conditions, no open channel event in $1 \mathrm{mM}$ ATP was observed. In contrast, open channel events could be identified clearly after irradiation. This result indicates that irradiation reduces the
ATP sensitivity. A quantitative measure of the ATP sensitivity change is presented as the half-inhibitory [ATP] $\left(\mathrm{IC}_{50}\right)$ and Hill coefficient $(H)$ (Table 2) from the fit of a Hill saturation function to the relationship between ATP inhibition and [ATP] (Figure 7). The maximal open probability and $\mathrm{IC}_{50}$ both increased significantly after irradiation (Table 2). The changes in these parameters might have been involved in the increased channel activity observed in both cell-attached and excised patches.

\section{Discussion}

In this study, we report that infrared low intensity laser irradiation activated KATP channels of Kir6.2-transfected 


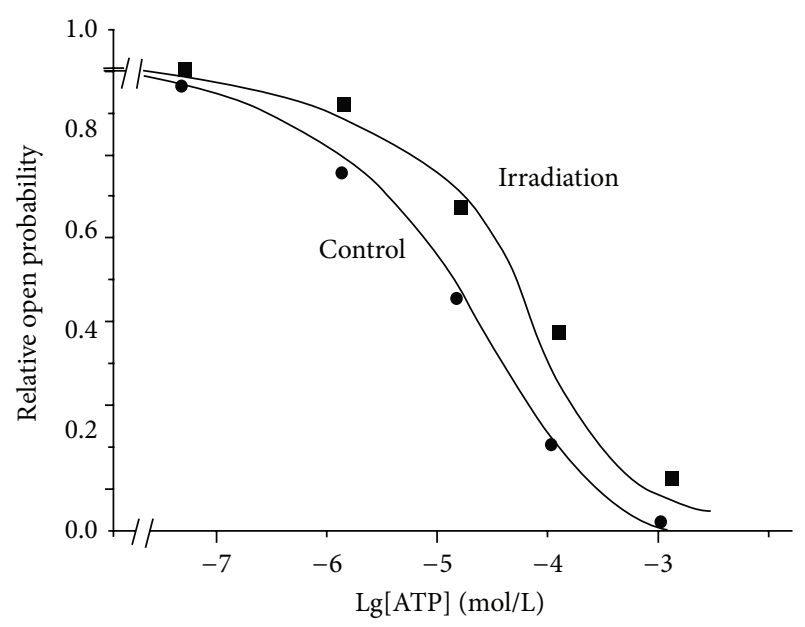

FIgURE 7: Activation of KATP channels by photobiomodulation. Effect of irradiation on the concentration-dependent inhibition of KATP channels by ATP. and a data collected before and after irradiation, respectively.

TABLE 2: Irradiation-induced activation of KATP channels.

\begin{tabular}{lccc}
\hline & $P_{\max }(\mathrm{NO}$ ATP $)$ & $\mathrm{IC}_{50}$ & $H$ \\
\hline Control & 0.204569 & $78.6 \pm 6.3$ & $1.1 \pm 0.2$ \\
Irradiation & $0.247436^{* *}$ & $103.7 \pm 9.2^{* *}$ & $1.2 \pm 0.2^{*}$ \\
\hline
\end{tabular}

${ }^{* *} P<0.01 ;{ }^{*} P<0.05$

$P_{\max }$ : maximal open probability. The concentration of ATP ([ATP]) producing half-maximal inhibition $\left(\mathrm{IC}_{50}\right)$ and the Hill coefficient $(H)$ were obtained by fitting the measured open probability data with the expression $P_{\mathrm{O}} / P_{\max }=$ $1-A^{H} /\left(A^{H}+\mathrm{IC}_{50}{ }^{H}\right)$.

HEK-293 cells and excised patches directly. A prominent increase in KATP channel activity was perceived during and after irradiation and the current amplitude improved (Figure 4). An experimental recording of the photobiomodulation on single KATP channels showed that a Kir6.2 channel was active from the beginning of the experiment when the excised patch was perfused without ATP and the open probability, current amplitude, and dwell time of KATP channel improved after the irradiation (Figure 5). Compared with the channels that did not undergo irradiation, the current amplitude and dwell time of KATP channel improved after irradiation (Table 2, Figure 6). In excised patches, the activation of a KATP channel can be dissected into three major components: (1) an increase in maximal open probability, (2) a decrease in ATP sensitivity, and (3) an increase in dwell time of a KATP channel. The reduced ATP sensitivity in patches excised from cells exposed to irradiation indicated a change in the intrinsic KATP channel sensitivity which is at least a partial cause of channel activation (Figure 4).

Using UV photoirradiation stimulation on KATP channels of rat heart and recombinant KATP channels composed of SUR2 and Kir6.2 in intact cells and in excised patches, it was found that the modification favoring channel opening triggered by UV photoirradiation affects KATP channel activity partly by regulating membrane phosphoinositide levels [4]. It was also found that free radical scavengers retarded irradiation induced activation of KATP channel [4]. As free radicals have been shown to increase the opening of cardiac KATP channels by increasing the open probability and reducing the ATP sensitivity [5-7], It was proposed that the upstream step that transforms UV irradiation energy into stimulation of enzymatic production of phosphoinositides is the production of ROS $[4,8]$. The results of our electrophysiological study on low intensity infrared laser irradiation on KATP channel are very similar with the results of Fan's electrophysiological study on UV irradiation on KATP channel. In order to interact with the living cell, light has to be absorbed by intracellular chromophores. There is a growing body of evidence that suggests cytochrome $\mathrm{c}$ oxidase is the primary photoacceptor of nucleated cells which can absorb the light energy [9-12]. Here, we infer that cytochrome c oxidase may play a similar role as UV irradiation in KATP channel. We propose that cytochrome $\mathrm{c}$ oxidase may absorb the photons from low intensity infrared laser irradiation, produce ROS, and thus stimulate the production of phosphoinositides, while our data do not totally exclude other effects such as direct structural modification of membrane KATP channels of neuron.

Many physical and chemical stimuli such as photoirradiation, photochemical treatment, and osmotic stress share an array of common or closely related signal cascades to evoke a cellular response. So our results have the similar physiological implications in terms of the response of KATP channels to other physical stimuli of cell stresses [13].

In this study, we only observed some interesting phenomena, and much more work like the relationship among infrared low intensity laser irradiation with changes in membrane phosphoinositide and KATP channels should be explored later.

The use of TLS which involves photobiomodulation on neurological functions has gained significant interest in recent years $[14,15]$. TLS has shown significant beneficial and sustained effects in animal stroke models [15] and human trial (the NEST-1 and the NEST-2) [16, 17] for stroke. It shows photobiomodulation may be an alternative intervention modality for ischemic stroke. It is well known that there exists an "ischemic penumbra" around the infarct area in the acute ischemic stroke. How to save the neuron in the cerebral ischemia penumbra area is the key to improve the cure rate of acute ischemic stroke. Membrane KATP channels are the marker of cellular energy metabolism which links the membrane potential to the metabolic state of the cell as reflected by the levels of nucleoside triphosphates and diphosphates. As it was reported that activation of KATP channels plays a protective effect on hypoxic ischemic neurons [18], the phenomena we observed that low intensity laser irradiation can active membrane KATP channels may help to reveal the protection mechanism of low intensity $808 \mathrm{~nm}$ laser irradiation on ischemic hypoxic neurons.

\section{Conclusions}

Low intensity $808 \mathrm{~nm}$ laser irradiation at $5 \mathrm{~mW} / \mathrm{cm}^{2}$ may activate membrane KATP channels of Kir6.2-transfected HEK293 cells and in excised patches. 


\section{Conflict of Interests}

The authors declare that there is no conflict of interests regarding the publication of this paper.

\section{Acknowledgments}

This study had the financial support of the National Natural Science Foundation of China (no. 61078071), the Natural Science Foundation of Shanghai (no. 09ZR1422500), the State Key Development Program for Basic Research of China (no. 2013CB91060101), and The 2013 "Innovation Action Plan" from Science and Technology Commission of Shanghai Municipality (no. 13DZ1940200).

\section{References}

[1] Q. Zhang, H. Ma, S. Nioka, and B. Chance, "Study of near infrared technology for intracranial hematoma detection," Journal of Biomedical Optics, vol. 5, no. 2, pp. 206-213, 2000.

[2] L. M. Konstantinovic, M. B. Jelic, A. Jeremic, V. B. Stevanovic, S. D. Milanovic, and S. R. Filipovic, "Transcranial application of near-infrared low-level laser can modulate cortical excitability," Lasers in Surgery and Medicine, vol. 45, no. 10, pp. 648-653, 2013.

[3] H. Yokoshiki, M. Sunagawa, T. Seki, and N. Sperelakis, "ATPsensitive $\mathrm{K}+$ channels in pancreatic, cardiac, and vascular smooth muscle cells," American Journal of Physiology-Cell Physiology, vol. 274, no. 1, part 1, pp. C25-C37, 1998.

[4] Z. Fan and R. A. Neff, "Susceptibility of ATP-sensitive K+ channels to cell stress through mediation of phosphoinositides as examined by photoirradiation," Journal of Physiology, vol. 529, part 3, pp. 707-721, 2000.

[5] K. Tokube, T. Kiyosue, and M. Arita, "Openings of cardiac KATP channel by oxygen free radicals produced by xanthine oxidase reaction," American Journal of Physiology-Heart and Circulatory Physiology, vol. 271, no. 2, part 2, pp. H478-H489, 1996.

[6] K. Tokube, T. Kiyosue, and M. Arita, "Effects of hydroxyl radicals on K(ATP) channels in guinea-pig ventricular myocytes," Pflugers Archiv European Journal of Physiology, vol. 437, no. 1, pp. 155-157, 1998.

[7] K. Ichinari, M. Kakei, T. Matsuoka, H. Nakashima, and H. Tanaka, "Direct activation of the ATP-sensitive potassium channel by oxygen free radicals in guinea-pig ventricular cells: its potentiation by MgADP," Journal of Molecular and Cellular Cardiology, vol. 28, no. 9, pp. 1867-1877, 1996.

[8] Y. Kabuyama, M. Hamaya, and Y. Homma, "Wavelength specific activation of PI 3-kinase by UVB irradiation," FEBS Letters, vol. 441, no. 2, pp. 297-301, 1998.

[9] N. R. Sims and M. F. Anderson, "Mitochondrial contributions to tissue damage in stroke," Neurochemistry International, vol. 40, no. 6, pp. 511-526, 2002.

[10] M. T. T. Wong-Riley, H. L. Liang, J. T. Eells et al., "Photobiomodulation directly benefits primary neurons functionally inactivated by toxins: role of cytochrome c oxidase," Journal of Biological Chemistry, vol. 280, no. 6, pp. 4761-4771, 2005.

[11] J. T. Eells, M. M. Henry, P. Summerfelt et al., "Therapeutic photobiomodulation for methanol-induced retinal toxicity," Proceedings of the National Academy of Sciences of the United States of America, vol. 100, no. 6, pp. 3439-3444, 2003.
[12] P. A. Lapchak and L. de Taboada, “Transcranial near infrared laser treatment (NILT) increases cortical adenosine- $5^{\prime}$-triph osphate (ATP) content following embolic strokes in rabbits," Brain Research, vol. 1306, pp. 100-105, 2010.

[13] T. C. Y. Liu, Y. Y. Liu, E. X. Wei, and F. H. Li, "Photobiomodulation on stress," International Journal of Photoenergy, vol. 2012, Article ID 628649, 11 pages, 2012.

[14] A. Oron, U. Oron, J. Chen et al., "Low-level laser therapy applied transcranially to rats after induction of stroke significantly reduces long-term neurological deficits," Stroke, vol. 37, no. 10, pp. 2620-2624, 2006.

[15] P. A. Lapchak, J. Wei, and J. A. Zivin, "Transcranial infrared laser therapy improves clinical eating scores after embolic strokes in rabbits," Stroke, vol. 35, no. 8, pp. 1985-1988, 2004.

[16] J. A. Zivin, G. W. Albers, N. Bornstein et al., "Effectiveness and safety of transcranial laser therapy for acute ischemic stroke," Stroke, vol. 40, no. 4, pp. 1359-1364, 2009.

[17] Y. Lampl, J. A. Zivin, M. Fisher et al., "Infrared laser therapy for ischemic stroke: a new treatment strategy-results of the NeuroThera Effectiveness and Safety Trial-1 (NEST-1)," Stroke, vol. 38, no. 6, pp. 1843-1849, 2007.

[18] X.-L. Sun and G. Hu, "ATP-sensitive potassium channels: a promising target for protecting neurovascular unit function in stroke," Clinical and Experimental Pharmacology and Physiology, vol. 37, no. 2, pp. 243-252, 2010. 

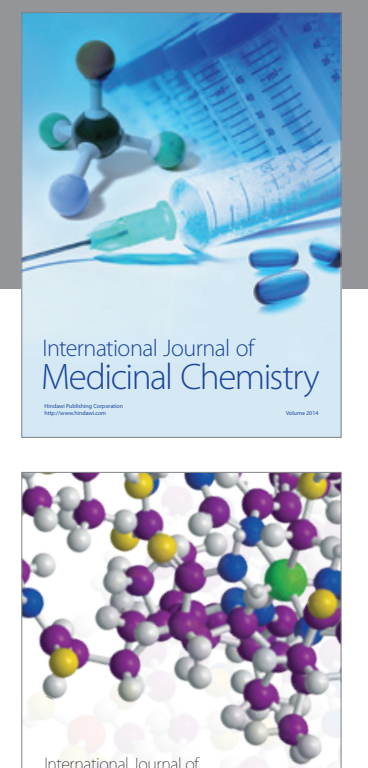

\section{Carbohydrate} Chemistry

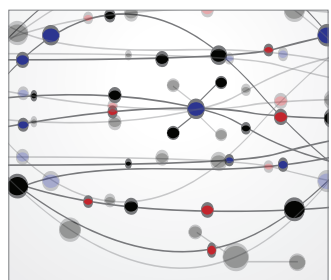

The Scientific World Journal
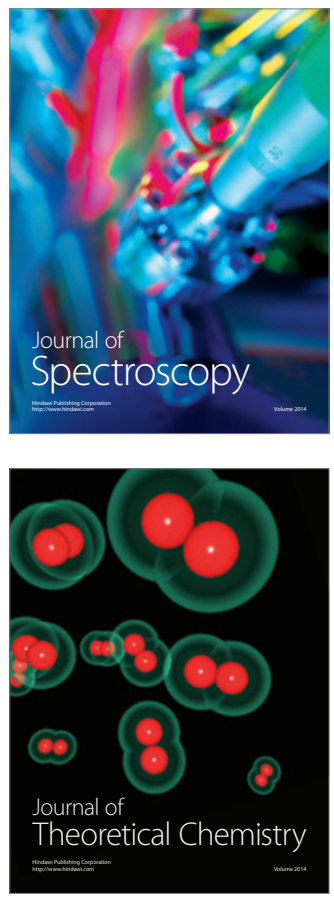
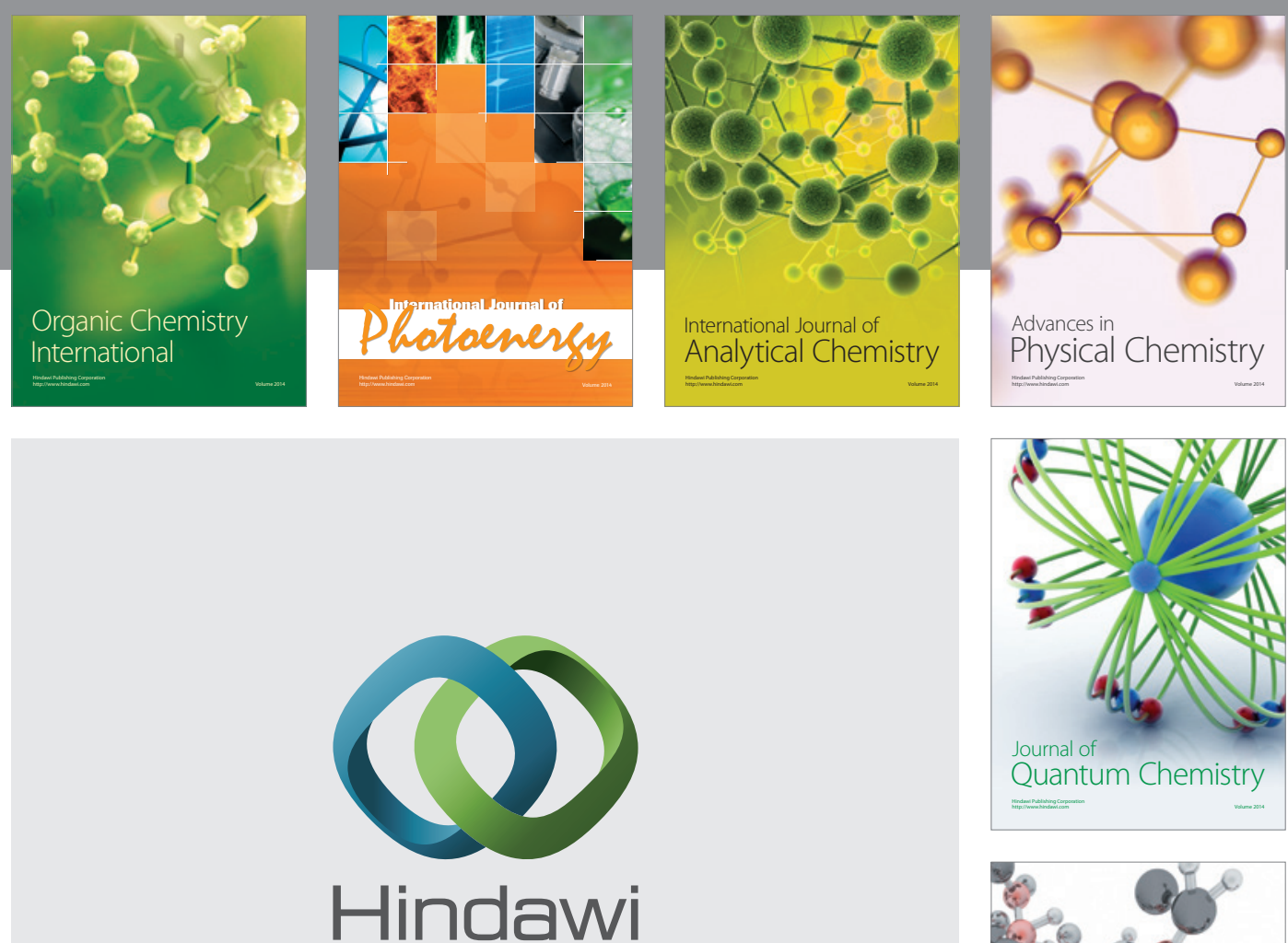

Submit your manuscripts at

http://www.hindawi.com

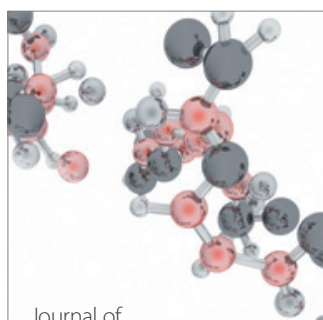

Analytical Methods

in Chemistry

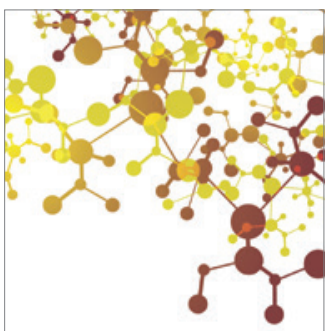

Journal of

Applied Chemistry

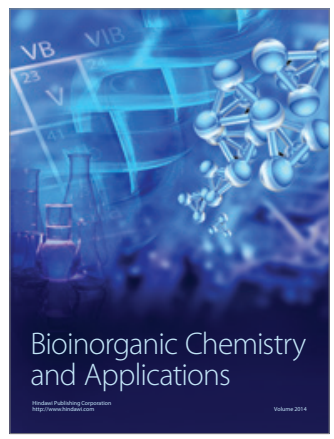

Inorganic Chemistry
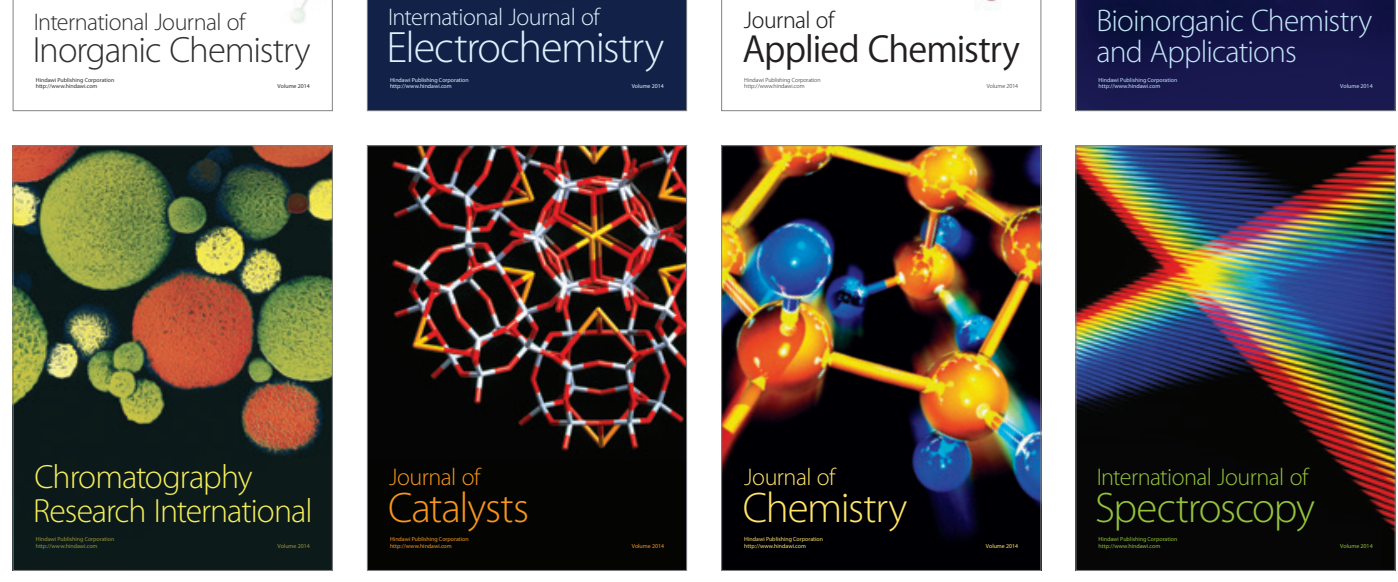